\title{
Actividades de aprendizaje y TIC: Usos entre docentes de la Educación General Básica costarricense. Aproximación diagnóstica
}

\author{
Learning Activities and ICT: Uses among Costa Rican Basic General Educators. \\ A Diagnostic Approach
}

\author{
Karolina Campos Jiménez ${ }^{\top}$ \\ Universidad Nacional \\ Heredia, Costa Rica \\ kcampos.jmnz@gmail.com \\ Vivian Carvajal Jiménez² \\ Universidad Nacional \\ Heredia, Costa Rica \\ vivian.carvajal@gmail.com \\ Enia Romilia Castro Gutiérrez ${ }^{3}$ \\ Ministerio de Educación Pública \\ San José, Costa Rica \\ enia.romilia@gmail.com \\ Sandra Hutchinson Heath ${ }^{4}$ \\ Ministerio de Educación Pública \\ San José, Costa Rica \\ sandra.hutch@gmail.com \\ Maribel Masís Muñoz \\ Ministerio de Educación Pública \\ San José, Costa Rica \\ mmasism@gmail.com \\ Susana Murillo León 6 \\ Universidad Nacional \\ Heredia, Costa Rica \\ smurilloleon@gmail.com \\ Grace Rojas Alvarado7 \\ Ministerio de Educación Pública \\ San José, Costa Rica \\ grace.maria@hotmail.com
}

1 Académica en el Instituto de Estudios Interdisciplinarios de la Niñez y la Adolescencia (UNA). Licenciada en Psicología y en Docencia.

2 Colaboradora en esta investigación. Académica de la División de Educación Rural (UNA). Filóloga y pedagoga. Doctora en Tecnología Educativa.

3 Licenciada en Educación Ambiental. Magister en Tecnología Educativa. Asesora Nacional de Educación e Investigadora en el Departamento de Investigación y Desarrollo Educativo del Instituto de Desarrollo Profesional Uladislao Gámez Solano (MEP).

4 Asesora Nacional. Ministerio de Educación Pública. Dirección de Recursos Tecnológicos en Educación, Dpto de Investigación, Desarrollo e Implementación. Docente de preescolar e informática educativa, máster en Administración Educativa.

5 Licenciada en Educación Especial, máster en Educación en Valores, Asesora Nacional de Educación en el Departamento de Investigación, Desarrollo e Implementación de la Dirección de Recursos Tecnológicos en Educación del Ministerio de Educación Pública.

6 Académica en la División de Educación Básica (UNA). Docente de inglés para l y ll ciclos. Máster en Artes en Enseñanza de Idiomas Internacionales.

7 Licenciada en Filosofía. Máster en Administración Educativa. Doctora en Educación. Asesora Nacional de Educación e Investigadora en el Departamento de Investigación y Desarrollo Educativo del Instituto de Desarrollo Profesional Uladislao Gámez Solano (MEP). 
URL: http://www.una.ac.cr/educare

CORREO: educare@una.cr

Fabián Rojas Ramírez ${ }^{8}$

Universidad Nacional

Heredia, Costa Rica

fabian.rojasr@gmail.com

Giovanny Sánchez Chacón ${ }^{9}$

Universidad Nacional

Heredia, Costa Rica

vanni80s@gmail.com

Recibido 11 de octubre de 2013 • Corregido 22 de noviembre de 2013 • Aceptado 06 de diciembre de 2013

Resumen. Dos de las entidades encargadas de administrar y decidir acerca de la formación inicial y continua de los docentes costarricenses, a saber, el Ministerio de Educación Pública (MEP) y la Universidad Nacional (UNA) ${ }^{10}$, desarrollaron entre 2011 y 2012 un proceso investigativo para apoyar la construcción de conocimientos relacionados con la incorporación de las tecnologías de la información y la comunicación (TIC) en contextos educativos y, al mismo tiempo, participar de la generación de innovaciones que coadyuven a fundamentar y orientar las políticas educativas nacionales y de formación docente. Dicha indagación parte del reconocimiento de actividades de aprendizaje que se apoyan en las TIC y que son implementadas por el personal docente, en la Educación General Básica (EGB), como una forma de orientar su quehacer educativo hacia espacios críticos, colaborativos y gestores de aprendizajes relevantes para los involucrados. Para esta exploración, se consideran catorce de las regiones educativas en las que el MEP divide el país: Desamparados, Limón, San Ramón, Los Santos, Pérez Zeledón, Grande de Térraba, Puntarenas, Aguirre, Coto, Liberia, Upala, Cañas, Santa Cruz y Nicoya. Para el abordaje teórico, esta propuesta se basa en los aportes de César Coll (2005), quien parte de las relaciones existentes en lo que este autor llama el triángulo interactivo; una metodología que remite al estudio de relaciones entre tres elementos: el contenido, la actividad educativa instruccional del profesorado y las actividades de aprendizaje del grupo estudiantil. En este artículo se abordan nueve de las categorías que Coll (2005) señala como frecuentes en las prácticas de mediación que involucran las TIC y, adicionalmente, emergen tres categorías que la investigación arroja para comprender en qué situaciones y con qué fines los docentes costarricenses de la EGB emplean las TIC durante su práctica pedagógica. Este trabajo es desarrollado por un equipo interinstitucional de investigadores de la Universidad Nacional y el Ministerio de Educación Pública.

Palabras claves. TIC, actividades de aprendizaje, Educación General Básica, triángulo interactivo.

8 Coordinador del equipo de investigación Perfiles (UNA). Académico de la División de Educología (CIDE). Especialista en Informática Educativa. Máster en Educación con mención en Docencia Universitaria.

9 Académicon en la División de Educología (UNA). Docente de educación musical, pedagogo y doctor en Psicología de la Educación, Cultura y Sistemas Semióticos.

10 Representados por el Departamento de Investigación, Desarrollo e Implementación (DIDI) y el Instituto Uladislao Gámez, en el caso del MEP; y por el Centro de investigación y Docencia en Educación (CIDE) y el Proyecto Perfiles y Dinámicas de la Educación Costarricense, en el caso de la UNA. 
Abstract. Between 2011 and 2012, two of the entities responsible for managing and deciding onthe initial and continuing education of Costa Rican teachers, the Ministry of Public Education (MEP) and the National University (UNA)"11, developed a research process to support the construction of knowledge related to the inclusion of Information and Communication Technologies (ICTs) in educational contexts, and at the same time, participate in the generation of innovations that help to create and guide national educational policies and teacher preparation. This research project is based on the recognition of learning activities supported by ICTs and implemented by teachers in the Basic General Education (EGB) as a way to guide their educational work towards critical and collaborative spaces that promote learning and are relevant to those involved. This study considered fourteen of the educational regions in which the MEP divides the country: Desamparados, Limón, San Ramon, Los Santos, Pérez Zeledón, Grande de Térraba, Puntarenas, Aguirre, Coto, Liberia, Upala, Cañas, Santa Cruz, and Nicoya. The theoretical framework is based on the contributions by César Coll (2005), who departs from the existing relationships in, what this author calls, the interactive triangle, a methodology that refers to the study of relationships between three elements: content, the teacher's instructional and educational activities, and the students' learning activities. This paper addresses nine of the categories Coll (2005) points out as frequent mediation practices involving ICTs. In addition, the research establishes three categories to understand the circumstances and the reasons why Costa Rican EGB teachers use ICTs during their pedagogical practices. This paper was developed by an interinstitutional team of researchers from the National University and the Ministry of Public Education.

Keywords. ICT, learning activities, Basic General Education, interactive triangle.

\section{Un acercamiento a la propuesta}

La introducción de las TIC en las aulas está poniendo en evidencia una nueva definición de roles, especialmente para los alumnos y docentes. Los primeros, gracias a estas nuevas herramientas, están adquiriendo mayor autonomía y responsabilidad en el proceso de aprendizaje, lo que obliga al docente a salirse de su rol clásico como única fuente de conocimiento. ... Esto genera incertidumbres y temores; realidad que obliga a una readecuación creativa de la institución escolar

(Lugo, 2010, p. 58)

En atención a las demandas educativas del país, el MEP y el CIDE buscan gestionar conocimientos que sirvan como base para acelerar las transformaciones requeridas en el ámbito educativo-tecnológico a través de un diagnóstico que facilite el análisis de actividades de aprendizaje que involucren las TIC y sean implementadas por los docentes en la Educación General Básica (EGB).

Represented by the Department of Research, Development, and Implementation (DIDI) and the Uladislao Gámez Institute, in the case
of MEP, and by the Center for Educational Research and Teaching (CIDE) and the Costa Rican Education Profiles and Dynamics Project, in the case of UNA. 
URL: http://www.una.ac.cr/educare

CORREO: educare@una.cr

El desarrollo de nuevos ambientes de aprendizaje incide en todos los actores curriculares, en particular sobre los docentes, quienes desde las nuevas condiciones de aprendizaje social se ven obligados a actuar como guías ante sus estudiantes $y$, de igual modo, gestionar recursos de aprendizaje que les faciliten a los discentes buscar, obtener, procesar, discernir, seleccionar y comunicar la información para convertirla en conocimiento útil y promotor de calidad de vida. En ese sentido, Salinas (1997, p. 10) manifiesta que las nuevas habilidades docentes tendrán "...como resultado implicaciones en su preparación profesional y se les va a requerir, en su proceso de formación -inicial o en ejecución-, a ser usuarios sofisticados de recursos de información ... de un amplio rango de herramientas de información y comunicación actualmente disponibles...".

Estos hechos deben valorarse también a la luz de otras situaciones y demandas del contexto y la realidad nacional, dado que el éxito o fracaso de la introducción de herramientas que impliquen procesos innovadores en las prácticas de mediación pedagógica se vinculan con todo un entramado de circunstancias, mitos, hábitos, necesidades, temores y percepciones (Claro, 2010).

Los argumentos anteriores son también coherentes con estudios realizados en ámbitos internacionales. De acuerdo con Coll, Mauri y Onrubia (2008), los usos relacionados con la comunicación y la colaboración son prácticamente inexistentes en las evidencias que se han hallado respecto del empleo que los docentes suelen darles a las TIC. Estos investigadores señalan que los usos que los docentes reportan para tales recursos remiten meramente a acciones que se sitúan en su espacio de trabajo personal (uso del procesador de textos, búsqueda de información en internet, preparación de clases, gestión del trabajo personal). Por otra parte, los menos frecuentes corresponden con los de apoyo a la labor docente en el aula (simulaciones, empleo de software educativo, presentaciones etcétera) y los vinculados a la comunicación y al trabajo colaborativo entre alumnos.

Este hecho nos plantea como imperiosa la necesidad de estimular prácticas de mediación que prioricen la construcción colaborativa del conocimiento, la participación ciudadana, la reflexión y el autoaprendizaje.

Sin duda, dentro del contexto de acceso a los beneficios de las TIC, Costa Rica tiene en sus manos un enorme potencial para desafiar la brecha digital, apoyar la formación inicial de educadores y enriquecer la actualización de los docentes en ejercicio con miras a generar una cultura escolar renovada, eficiente y de calidad.

No obstante, es vital que toda propuesta de innovación surja desde los propios actores, desde sus parámetros, realidades y demandas, pues su involucramiento genuino no solo le da mayor rigurosidad y confiabilidad al trabajo, sino que influye como factor motivacional sobre los participantes.

En Costa Rica, los cambios en el rol del docente en cuanto al uso de las TIC se han dado a pasos desiguales. Nuestras propuestas formativas demandan del educador gran conocimiento de las áreas temáticas de especialización, conocimiento didáctico y pedagógico general, pero 
ahora, además, el uso, acceso y apropiación de las TIC. Aunque algunos centros educativos han incursionado en el empleo de los recursos, otros apenas inician esfuerzos, ocasionalmente de manera empírica, sin la fundamentación teórica, metodológica y práctica que justifique el uso de las TIC como herramientas para la enseñanza y el aprendizaje.

Así, el objetivo central que se expone en este artículo, dicta: Analizar las prácticas didácticas mediadas con TIC en docentes de EGB de 14 regiones educativas de Costa Rica. Desde dicho fin, las TIC se visualizan como un instrumento para potenciar las capacidades de aprendizaje y sus usos en el espacio conceptual del triángulo interactivo propuesto por Coll, Mauri y Ornubia (2008); exposición que el equipo investigador acoge no solo por su claridad y consistencia epistemológica, sino porque se trata de una teoría validada y de alta confiabilidad.

Es importante anotar, sin embargo, que además de rectificar la propuesta de Coll (2004) -a excepción de una de las categorías-, el equipo investigador de este trabajo propone tres nuevas tipologías surgidas durante el proceso investigativo, contribuyendo con ello al acervo científico alrededor de la temática.

\section{Un acercamiento a la teoría: usos de las TIC en el espacio conceptual del triángulo interactivo}

Las aportaciones realizadas por Coll (2004) señalan algunos de los usos de las TIC en contextos y espacios educativos formales, en donde simultáneamente se propone una clasificación acerca de los usos que estas adoptan dentro del espacio conceptual del triángulo interactivo. Este documento aborda nueve de las diez categorías que Coll expone, además de tres que emergen en el marco de esta investigación, como usos de las TIC presentes en las prácticas de mediación de los docentes de EGB.

\section{Contenidos de aprendizaje (CA)}

Las TIC ocupan el vértice del triángulo interactivo correspondiente a los contenidos. Es el caso de los procesos educativos orientados a promover el aprendizaje del funcionamiento de las computadoras, de sus utilidades y aplicaciones; de las características y utilización de internet; del manejo de redes de trabajo con ordenadores, entre otros. Según lo refieren Bustos y Coll (2010, p. 166), las TIC “...se incorporan como contenidos específicos de enseñanza y aprendizaje ...con el objetivo fundamental de enseñar a usar las computadoras, el software específico e incluso las características del hardware".

Sobre este particular, se puede aducir que la actuación docente es influida por la necesidad de que el estudiantado conozca y maneje las herramientas tecnológicas de previo a incorporar su utilización en proyectos y tareas que conduzcan a aprendizajes significativos relacionados con contenidos propios del currículo. 
URL: http://www.una.ac.cr/educare

CORREO: educare@una.cr

Así se explica que, a pesar de que el plan de estudios de cada nivel educativo carece de una designación de tiempo para esos aprendizajes técnicos, los docentes lo disponen en la planificación de las actividades por realizar cotidianamente con los estudiantes. Puede agregarse que, tal como lo señalan Coll, Mauri y Onrubia (2008), los docentes sienten la necesidad de invertir tiempo en la enseñanza de los programas para conseguir más tarde que se incorpore su uso en los contenidos de aprendizaje que interesan en el currículo oficial.

\section{Repositorios de contenidos de aprendizaje (RCA)}

De acuerdo con Coll (2004), en esta categoría se agrupan aquellas experiencias en las que se utilizan las TIC para almacenar, organizar y facilitar el acceso de profesores y estudiantes a los contenidos. Los repositorios pueden ser más o menos completos, en el sentido de que pueden incluir la totalidad de los contenidos o solo una parte de ellos. También pueden ser abiertos -cuando incluyen accesos a otros repositorios de contenidos- o cerrados. Los cursos en línea en los que una parte o la totalidad del material de trabajo está "colgado en la red" son un ejemplo de este tipo de uso.

\section{Herramientas de búsqueda y selección de contenidos de aprendizaje (HBSCA)}

De acuerdo con Coll (2004), las TIC se asocian a la búsqueda, exploración y selección de contenidos de aprendizaje relevantes y apropiados a un determinado ámbito de conocimiento o de experiencia. En el área pedagógica, este uso se suele vincular con metodologías de enseñanza y aprendizaje basadas en casos o problemas; y respecto a lo tecnológico, a recursos de navegación y de exploración de bases de datos.

La búsqueda de información confiable es una destreza importante para los estudiantes, y para ello, la internet constituye una excelente herramienta para aprender de modo independiente o para obtener materiales de todo tipo con los cuales realizar las tareas escolares.

Desde esta perspectiva, Picardo (2002) recalca que los procesos de enseñanza y aprendizaje, desde la acción docente, implican facilitarles a los estudiantes el acceso a nuevos conocimientos utilizando variadas estrategias didácticas de información, tales como internet, libros, artículos de revistas y periódicos, enciclopedias electrónicas y físicas, entre otros.

\section{Herramientas de comunicación entre los participantes (HCP)}

De acuerdo con Coll (2004), estos usos potencian y extienden los intercambios comunicativos entre los participantes, estableciendo entre ellos auténticas redes y subredes de comunicación. A su vez, se utilizan recursos idénticos o diferenciados para la comunicación entre el profesorado y estudiantado y para la comunicación de estudiantes entre sí. Los mismos autores afirman que los recursos pueden estar diseñados con el fin de permitir una comunicación unidireccional o bidireccional, de uno a todos, de todos a uno, o de todos a todos. De la misma manera, dicha comunicación podría establecerse en tiempo real (sincrónica) o en distintos momentos (asincrónica). 
Para Coll (2004), algunos recursos tecnológicos o tecnológico-didácticos frecuentemente asociados a este uso son el correo electrónico, los grupos de noticias, las listas de distribución, los foros, los tableros electrónicos, los chats, las audioconferencias y las videoconferencias, entre otros. Así, los docentes también realizan distintos usos de las herramientas web $2.0^{12}$, especialmente el Facebook, como instrumentos psicológicos.

En concreto, las TIC, por las múltiples posibilidades que ofrecen, representan una plataforma interactiva de mucho valor para el desarrollo de espacios de comunicación que configuren nuevas formas de enseñanza y aprendizaje.

\section{Instrumentos cognitivos a disposición de los participantes (ICP)}

De acuerdo con Coll (2004), las TIC se utilizan fundamentalmente como instrumentos mediadores de la interacción entre estudiantes y contenidos, con el fin de facilitarles, a los primeros, el estudio, memorización, comprensión, aplicación, generalización o profundización de los segundos. Este uso suele estar asociado tanto a metodologías de enseñanza y aprendizaje basadas en la ejercitación y la práctica, como a metodologías orientadas a la comprensión; y desde el punto de vista tecnológico y didáctico, a recursos de realimentación, de navegación, de exploración de relaciones, de scaffolding (plantillas, ayudas...) y a la utilización de tecnologías y formatos multimedia e hipermedia.

La utilización de estos recursos permite que el estudiantado reelabore la información y la transforme en aprendizajes útiles. El papel del personal docente es vigilar que los participantes delimiten y filtren la información pertinente para los propósitos de aprendizaje, lo cual se refleja en todos los aspectos de la vida.

\section{Auxiliares o amplificadores de la actuación docente (AAD)}

Las TIC se utilizan fundamentalmente como herramientas que le permiten al personal docente apoyar, ilustrar, ampliar o diversificar sus explicaciones, demostraciones o actuaciones en general. Algunos ejemplos son el uso de internet o de un CD en el aula para ilustrar una explicación o apoyarla con la presentación de imágenes, documentos, esquemas, gráficos o simulaciones, entre otros. En relación con los datos anteriormente expuestos, Coll (2004) considera las TIC como instrumentos mediadores de la actividad conjunta desplegada por educadores y alumnos durante la realización de tareas o actividades de enseñanza y aprendizaje. Algunos ejemplos habituales de esta categoría son la utilización de las TIC como:

12 Se llama así al fenómeno social que implicó la aparición de una serie de aplicaciones que se valen del internet y que interpelan a la participación de usuarios activos, en constante interacción e interactividad. 
URL: http://www.una.ac.cr/educare

CORREO: educare@una.cr

- Auxiliares o amplificadores de determinadas actuaciones del profesorado (explicar, ilustrar, relacionar, sintetizar, proporcionar retroalimentación, entre otros).

- Comunicadores de valoraciones, críticas y otros, mediante el uso de presentaciones, simulaciones, visualizaciones o modelizaciones.

- Auxiliares o amplificadores de determinadas actuaciones del alumnado (hacer aportaciones, intercambiar informaciones y propuestas, mostrar los avances y los resultados de las tareas de aprendizaje...).

- Puentes para el seguimiento de los avances y dificultades de los alumnos.

- Seguimiento del propio proceso de aprendizaje de los alumnos.

- Mecanismo para solicitar u ofrecer realimentación, orientación y ayuda relacionada con el desarrollo de la actividad y sus productos o resultados.

En síntesis, se puede decir que las TIC son herramientas utilizadas para pensar, aprender, representar y transmitir los conocimientos, destrezas y valores adquiridos.

\section{Sustitutos de la acción docente (SAD)}

La actuación docente es también asumida por las TIC, ya que estas les proporcionan a los estudiantes los contenidos y las pautas para la realización de las actividades previstas para su aprendizaje y evaluación. Los tutoriales y los materiales educativos multimedia autosuficientes son ejemplos de este tipo de uso.

Esta categoría hace referencia a aquellos elementos que el docente pueda gestionar, crear o buscar para que el aprendizaje sea dirigido o favorezca el trabajo autónomo. De acuerdo con Monereo y Romero (2008, p. 210), es tarea del docente "...influir para que esos desarrollos y aplicaciones respondan a unas determinadas concepciones de lo que se debe aprender, enseñar y vivir en una sociedad justa y democrática".

De esta manera, los sustitutos de la acción docente son herramientas que promueven la autonomía, la creatividad y la metacognición, desarrollando en el estudiantado la capacidad para construir conocimiento.

\section{Instrumentos de evaluación de los procesos de enseñanza y aprendizaje (IEPEA)}

Este uso puede referirse al seguimiento del proceso de aprendizaje de los estudiantes y a su regulación por parte del profesorado; al seguimiento y autorregulación que los alumnos hacen de su propio proceso de aprendizaje; o al seguimiento y regulación tanto del proceso de aprendizaje de los alumnos como de la actuación docente del profesor (Coll, 2004). 
Desde el punto de vista tecnológico o tecnológico-didáctico, los recursos técnicos asociados a este uso suelen ser similares a los de otros usos de las TIC (como instrumentos de seguimiento y control, como instrumentos de evaluación de los resultados, como herramientas de comunicación y colaboración entre los participantes). El personal docente puede utilizar instrumentos de evaluación que respalden los procesos y productos elaborados por los estudiantes y que evidencien el logro o no de los aprendizajes esperados a través del trabajo individual, en equipo y por grupo.

La evaluación busca la valoración ética de cada participante, pues permite tomar conciencia del nivel de logro personal y colectivo. Además, establece la autorregulación de las actuaciones y del avance de estudiantes y educadores. La evaluación busca la obtención de conocimiento para tomar decisiones y proponer mejoras a los procesos de enseñanza y aprendizaje.

\section{Instrumentos de evaluación de los resultados del aprendizaje (IERA)}

Las TIC se utilizan para establecer pruebas o controles de los conocimientos o de los aprendizajes construidos por los estudiantes, y pueden situarse en diferentes momentos del proceso de aprendizaje: al inicio, al final o en puntos intermedios. Pueden ser pruebas o controles de heteroevaluación, autoevaluación o coevaluación, y adoptar formatos diversos: preguntas cerradas de elección múltiple, preguntas de sí o no, preguntas abiertas con espacio limitado de respuesta, elaboración de esquemas, definición de términos, resolución de problemas, ensayos de extensión y complejidad variable, entre otros. Asimismo, pueden ir acompañados de una realimentación que puede ser o no automática y más o menos inmediata.

Las TIC pueden presentarse en procesos de evaluación de diversas formas. Por ejemplo, el estudiante puede crear documentos que serán objetos de evaluación, como los textos cooperativos (para ello, se pueden emplear herramientas como Google Docs, Wikispaces). Otra manera es que la información sea enviada al profesor utilizando los medios disponibles (correo) o que comparta sus productos con recursos Web 2.0. Esto favorece una evaluación continua de manera formativa. Por otra parte, está la creación de mapas conceptuales como un recurso para la evaluación intermedia o final. Con ellos, el estudiante puede identificar conceptos importantes y sus relaciones en un campo particular de conocimiento.

Hasta aquí se exponen las tipologías ofrecidas por Coll (2004) para identificar el uso y la implementación que realizan los docentes de las tecnologías digitales. La investigación de marras ofrece otras tipologías emergentes sustentadas en el trabajo de campo, el aporte de los docentes, de expertos y del propio equipo investigador, así como la teoría contemporánea relacionada con las temáticas en estudio. A continuación se esbozan tres tipologías emergentes del proceso indagatorio. 
URL: http://www.una.ac.cr/educare

CORREO: educare@una.cr

\section{Potenciadores de las capacidades de aprendizaje (PCA)}

Los potenciadores de las capacidades de aprendizaje son las estrategias didácticas con el uso de TIC, implementadas por el docente para promover el desarrollo de capacidades de aprendizaje tales como: desarrollo de la creatividad, aprendizaje significativo, pensamiento estratégico, pensamiento lógico-matemático, entre otros.

Las TIC representan un campo de acción de mucho valor para el desarrollo de procesos creativos en los estudiantes (Coll, s. f.). Las múltiples posibilidades creadoras, de representación de la información y de interactividad que estas herramientas posibilitan las convierten en un escenario prominente para el desarrollo de procesos creadores que repercutan en la concepción de nuevas formas de enseñar y aprender.

De acuerdo con Pozo, Monereo y Castelló (2001), el uso del conocimiento estratégico implica una toma de decisiones acerca de cuándo, cómo, por qué o en qué condiciones hacer uso de unos determinados procedimientos para aproximarse a una meta de aprendizaje. Desde esta perspectiva, el conocimiento estratégico está vinculado al desarrollo de habilidades metacognitivas y de autorregulación del aprendizaje que, de manera progresiva, fomentan la autonomía en dicho proceso.

Las TIC representan instrumentos de grandes posibilidades para favorecer estrategias de enseñanza y aprendizaje que fomenten un uso más consciente del conocimiento estratégico, lo cual tendrá un impacto seguro en la formación de aprendices permanentes, capaces de instruirse a lo largo de la vida y transferir lo aprendido a diversas situaciones y contextos.

\section{Producción de objetos tecnológicos con tecnología (POTT)}

Los objetos tecnológicos son aquellos que los seres humanos han desarrollado como respuesta a una necesidad, con el fin de facilitar las tareas cotidianas y mejorar su calidad de vida.

Como categoría emergente de este estudio, la tipología sobre la producción de objetos tecnológicos por medio de tecnología (POTT) se conceptualiza como la utilización de las TIC para producir objetos por medio de otras tecnologías aprendidas por los estudiantes. Estos productos se reflejan en procesos de investigación, diseño, programación y producción de objetos como posibles soluciones a problemas contextualizados a su entorno. Algunos ejemplos de estos productos son aquellos creados con software de robótica y mecanismos robóticos, juegos programados con software especializado, libros interactivos y multimedios.

Esta tipología es clasificada en dos tipos: a) Producción de tecnologías para resolución de problemas y b) Diseño de recursos didácticos que favorecen el aprendizaje. 


\section{Aspectos emocionales y el uso de TIC (ASE)}

Con frecuencia, la educación tradicional centra sus iniciativas en el desarrollo de aspectos cognitivos de los estudiantes, lo cual, entre otras cosas, ha causado el abandono de la enseñanza explícita de otras competencias de interés que podrían también abordarse en la escuela, como lo es la educación socioafectiva y los aspectos emocionales (Pérez-González, 2012).

Las reacciones emocionales son naturales en el ser humano: mantienen la curiosidad, materializada a través del interés que despierta descubrir algo nuevo, ya sea una actividad o aprendizaje. Es importante recalcar la relevancia que esto cobra para la labor docente, ya que la atención de los estudiantes se puede evocar por medio de la curiosidad, elemento que se describe como indisoluble de la emoción.

Desde esta perspectiva, es relevante enfatizar en que las emociones constituyen un lenguaje que nos permite comunicarnos con los otros, pudiendo facilitar o dificultar la transmisión de conocimientos.

Quizá uno de los factores más importantes que inciden en la autorregulación del aprendizaje y de las emociones que se desencadenan en dicho proceso, es el papel de la motivación, pues esta permite el establecimiento de un contexto intrasicológico favorecedor de estados emocionales que propician el aprendizaje.

De esta forma, también sería importante señalar otro principio relacionado con la autenticidad de los procesos de enseñanza y aprendizaje (Monereo, Castelló y Gómez, 2009) donde se establece la importancia de que todo proceso educativo procure de forma paralela la enseñanza de contenidos en contextos reales/situados de aplicación. Lo anterior, sin duda, permitiría valorar lo aprendido y atribuirle de manera más inmediata un sentido y aplicabilidad real.

Es importante reconocer que los principios señalados anteriormente juegan un papel muy importante en todos los aspectos de la vida, así como en la puesta en marcha de cualquier proyecto, especialmente si este tiene lugar en un entorno escolar.

Otro de los factores con un fuerte componente emocional y al que la investigación especializada le atribuye efectos directos sobre el aprendizaje, es el autoconcepto académico.

El autoconcepto, con toda la base emocional que sobre este se teje, posee implicaciones notables en el aprendizaje de un sujeto. Sin duda, influye considerablemente en las formas de autorregulación que el sujeto ejerce para aprender una determinada disciplina en contextos situados de enseñanza y aprendizaje.

En esta autorregulación entran en juego una gran cantidad de variables que impulsan o ralentizan el aprendizaje de un sujeto. Muchos de estos rasgos son de carácter motivacionalemocional, e influyen directamente en las diversas formas de autorregulación que un aprendiz emplea, tanto consciente como inconscientemente. 
URL: http://www.una.ac.cr/educare

CORREO: educare@una.cr

Con base en lo indicado, se puede reflexionar acerca de la necesidad de adoptar el uso cotidiano de las TIC en las aulas para captar la atención de los niños y adolescentes que están ya acostumbrados a interactuar, comunicar e inclusive aprender por medio de TIC.

Esto implica un reto para los sistemas educativos actuales, pues el ideal es la formación integral de los niños y adolescentes, incluyendo competencias emocionales que les permitan una mejor adaptación al contexto (Pérez-González, 2012). Para ello, es necesario que la educación contemple los cambios sociales del momento y utilice nuevas posibilidades -entre las que destacan las TIC-, principalmente por su implicación en la comunicación y las relaciones interpersonales.

\section{Cómo se aborda la metodología}

Investigar sobre la apropiación de los recursos tecnológicos en la práctica pedagógica desde la visión de los participantes significa ver a través de los ojos de los otros y las otras, buscando la manera de conocer la cotidianidad, las vivencias, las prácticas docentes, la diversidad y complejidad que comparten los sujetos actuantes.

Los informantes que colaboran con este estudio son 47 profesores de la EGB que participan en 14 talleres y actividades llevadas a cabo en 14 direcciones regionales de educación del país.

Para la selección de los participantes se solicita colaboración formal ante diversas asesorías regionales del MEP, con el propósito de identificar por cada sede regional, docentes que involucraran en el uso de tecnología en su práctica pedagógica y que laboren en instituciones donde se implementen proyectos relacionados con el uso de la tecnología. Dicha población debía haber implementado su proyecto entre 2010 y primer semestre de 2011, y pertenecer a centros educativos públicos de I, II y III ciclos de la EGB.

Las personas participantes pertenecen a las Regiones Educativas de Desamparados, Limón, San Ramón, Los Santos, Pérez Zeledón, Grande de Térraba, Puntarenas, Aguirre, Coto, Liberia, Upala, Cañas, Santa Cruz, y Nicoya. Para este estudio se tomó en consideración a participantes pertenecientes a 24 escuelas y 23 colegios. Como instrumentos de indagación se emplearon cuestionarios, autoinformes y fichas técnicas.

\section{Los resultados}

Tras el análisis de la información recopilada, destaca que entre un 2 y un $81 \%$ de las categorizaciones mencionadas reportan algún uso.

Respecto de la categoría contenido de aprendizaje (CA), los docentes que participan en la fase de diagnóstico del proyecto de marras muestran preferencia por el manejo de herramientas computacionales y la utilización de la web cuando incorporan la tecnología a las 
prácticas didácticas. Un 75\% de los docentes que aportan información en este sentido (28 en total) se inclinan hacia esa subcategoría.

Se refleja, además, un aprovechamiento de herramientas de la paquetería básica de Office, especialmente Word y Power Point, así como software para elaborar videos (Movie Maker, por ejemplo). En cuanto al uso de la web, se evidencia la predilección por sitios gratuitos y de información específica, como son las bibliotecas virtuales. También se inclinaron por acciones referidas a la apertura de cuentas de correo electrónico, programación en Robolab y elaboración de presentaciones multimedia en línea con la herramienta Prezi.

Asimismo, se puede colegir que sus prácticas didácticas incluyen en menor medida lo relativo al hardware y el uso de otros dispositivos, pues un $25 \%$ de la población docente que se incluye en esta categoría (7 docentes de 28 que reportan la presencia de la categoría, entre un total de 162 diagnosticados), otorga tiempo lectivo planificado explícitamente para realizar actividades que le permitan al estudiantado dominio del hardware y otros dispositivos. De esos, 3 se desempeñan como docentes de informática. Lo anterior evidencia como necesidad dedicar tiempo lectivo a la enseñanza del hardware y otros dispositivos.

Se puede aducir que parte de la actuación docente se ve influida por la necesidad de que el estudiantado conozca y domine las herramientas tecnológicas antes de incorporar su utilización en proyectos y tareas que conduzcan a aprendizajes significativos relacionados con contenidos propios del currículo.

Así se explica que, a pesar de que en el plan de estudios de cada nivel educativo no se designa tiempo horario a esos aprendizajes, los docentes lo otorgan en la planificación de las actividades por realizar cotidianamente con los estudiantes.

Así, los docentes indican su necesidad de enseñarles a los estudiantes el manejo instrumental de programas de apoyo general, tales como: procesadores de texto, elaboración de presentaciones multimedia, creación de videos y otros específicos, como Robolab y apertura de cuentas de correo electrónico. La mayoría de los docentes que brindan información en esta categoría, un 75\% (21 docentes), dirigen esfuerzos a realizar acciones y actividades relacionadas con la enseñanza de software como herramienta computacional, de manera que el estudiantado incorpore esos conocimientos en sus prácticas educativas y de vivencia general. Así, utilizan el procesador de textos Word, los creadores de presentaciones multimedia PowerPoint y Prezi, y las plataformas específicas de correo electrónico.

Para la categoría repositorio de contenido de aprendizaje (RCA), 3 docentes de un total de 47 ofrecen información. En esa misma línea se puede señalar que los datos permiten la subcategorización en dos vertientes: "creación de dispositivos digitales informativos en la web", en la que un dos de los docentes indican tener experiencias y, "creación de dispositivos digitales informáticos", donde un solo docente, realiza actividades con los estudiantes. 
URL: http://www.una.ac.cr/educare

CORREO: educare@una.cr

Los tres docentes manifiestan haber creado boletines, vídeos y folletos en torno a la asignatura o especialidad que imparten, al igual que han incursionado en la creación de blogs y páginas web para que el estudiantado acceda a los contenidos programáticos que se abordan en clase. Dos de estos educadores utilizan la tecnología para realizar creaciones digitales de carácter informativo con los estudiantes, incursionan en la elaboración de recursos propios que incluyen videos, boletines, blogs y otros que les permiten ampliar y reforzar los contenidos programáticos.

Por su parte, la categoría herramientas de búsqueda y selección de los contenidos de aprendizaje (HBSCA) evidencia usos en un total de 16 participantes (34\% del total de 47 datos aportados por los docentes participantes): indagación y selección de contenidos de aprendizajes, descarga de datos en la web, y búsqueda y selección de datos como insumo la su labor profesional.

La subcategoría que presenta mayor frecuencia en este grupo de 16 datos aportados es la que corresponde a la "indagación y selección de contenidos de aprendizaje, con un $68.8 \%$ de los datos, con lo que supera en más de un $40 \%$ a las otras dos subcategorías. En esta se enfatiza la búsqueda de información que realizan los estudiantes utilizando las TIC; pero no solo se queda en la mera búsqueda, sino que, debido a que los documentos o contenidos recopilados pueden ser también seleccionados, valorados, analizados o sintetizados por los estudiantes con la intención de utilizarlos en sus trabajos o en diferentes actividades de clase, estas competencias y destrezas son también puestas en práctica.

Entre las actividades que el estudiantado realiza con la información localizada se destacan la creación de diapositivas, exposiciones, proyectos, resúmenes, investigaciones y análisis, entre otros; para esto hacen uso del internet a través de la computadora o de dispositivos móviles.

La siguiente subcategoría es representada por 4 datos (individuos), con una frecuencia del $25 \%$, y corresponde con el uso de las "herramientas de búsqueda y selección de los contenidos de aprendizaje para descargar datos en la web". Se destaca porque la búsqueda de información tiene la finalidad de capturar imágenes, canciones y música de sitios de internet, con el propósito de que los estudiantes los utilicen en las actividades de aprendizaje. Se destaca el uso de diapositivas, la impresión de la información y el compartir ésta con los compañeros.

Por último, se encuentra la subcategoría "búsqueda y selección de datos por parte del docente como insumo para su labor profesional", en la cual identifica solo un dato, por lo que su frecuencia es de apenas 6.3\%. En esta se destaca como único dato que el docente explora en internet básicamente para obtener apoyos como fotografías y videos.

En este ámbito, es necesario destacar la importancia del uso de las TIC para la actualización del docente respecto a los contenidos por impartir y la utilización del internet y los recursos multimedia como material a su disposición para el desarrollo de la práctica pedagógica; situación prácticamente ausente de los usos reportados por los participantes en esta indagación. 
Por otro lado, para la categoría herramientas de comunicación entre los participantes (HCP), responden 16 de los 47 participantes (34\%). Aquí, se nota una predominancia de elementos vinculados a la subcategoría: Orientación comunicativa de las herramientas web 2.0", en la cual existen 15 respuestas vinculadas, lo que representa un $93,7 \%$ del total de quienes reportan este uso. Los docentes emplean diversas aplicaciones como herramientas de comunicación con sus estudiantes: el Facebook, blogs, chats, foros etcétera; pues permiten establecer vías de comunicación sincrónica y asincrónica, favoreciendo las potencialidades didácticas de las actividades de mediación que ellos emplean. De acuerdo con los datos, las prácticas más frecuentes de los docentes fueron el Facebook como herramienta de comunicación, los foros de discusión y los blogs.

En cambio, la subcategoría "orientación comunicativa a través de dispositivos móviles", contabiliza tan solo una respuesta, lo que representa apenas un $6,3 \%$ del total. En resumen, los datos encontrados indican que los docentes participantes en este estudio, si bien solo emplean las TIC como herramienta comunicativa en un 9,88\% de los casos, cuando lo hacen, con mayor frecuencia es como "herramientas de comunicación entre los participantes", a través del uso de las herramientas Web 2.0.

Para la quinta categoría, instrumentos cognitivos a disposición de los participantes (ICP), responden 13 del total de participantes (28\%). Para esta se tienen dos subcategorías. La primera, "instrumentos de mediación a partir de dispositivos móviles", representa un 92,3\% (12) de los datos, lo que indica que los docentes utilizan este tipo de aplicaciones para el desarrollo cognitivo de los estudiantes. Al considerar la información presentada es evidente que los docentes se inclinan hacia el uso de instrumentos de mediación a partir de software multimedia y aplicaciones interactivas; el del PowerPoint y el Geogebra son los que más destacan en esta categoría.

Estas prácticas educativas mediadas por TIC se dan en la interactividad tecnológica en las que se visualiza la relación "profesor-alumno-contenidos" (frases de los docentes anteriormente expuesta en las que, por medio de juegos multimedia y un programa de karaoke en la computadora, se aborda el aprendizaje del idioma inglés); como en la interactividad pedagógica, ejemplificada en el uso del PowerPoint como una "ayuda educativa" para que los estudiantes expongan algún contenido.

Como se ve, es limitada la cantidad de herramientas utilizadas por los docentes, pues en su mayoría son aplicaciones ofimáticas de presentación que solicitan la incorporación de elementos multimedia (texto, imágenes, video, audio) y en algunos casos, el uso de aplicaciones como Geogebra que, aunque no posibilita la integración de diferentes medios, les permite a los estudiantes manipular la información y, de esa forma, visualizar con mayor facilidad el comportamiento de datos y gráficos.

La segunda subcategoría es reportada por solo un participante $(7,7 \%)$, instrumentos de mediación a partir de dispositivos móviles; lo que indica que el uso de estos dispositivos en las aulas se explora de forma muy incipiente. 
URL: http://www.una.ac.cr/educare

CORREO: educare@una.cr

La sexta categoría, auxiliares o amplificadores de la actuación docente (AAD), es apoyada con evidencias de 15 de los participantes (32\%). En ella, la primera subcategoría es la relacionada con el empleo de recursos multimedia, la cual obtiene un 66,7\% (10 participantes), lo que indica que parte del personal docente que participa en este estudio realiza un importante esfuerzo por utilizar la tecnología como medio para dinamizar la práctica pedagógica. En este caso particular, mediante la utilización del proyector de multimedia (video beam).

La segunda subcategoría es la relacionada con el uso del software educativo, la cual obtiene 20\% (3 participantes). Uno de los educadores utilizó un software para el diseño y planificación de una investigación. Otro lo utilizó con guías de trabajo para que los estudiantes realizaran los ejercicios o actividades. Y, finalmente, la tercera subcategoría, a través de herramientas en línea, representa un 13,3\% (2 participantes) de los involucrados en este trabajo; una de ellas mediante un blog y el otro con un sitio web que trabaja con programación en Acrobat Flash.

En este sentido, es importante acotar que la implementación de programas educativos ofrece grandes posibilidades para la mediación pedagógica, ya que promueve el aprendizaje autónomo, la creatividad, la curiosidad, el interés, y la motivación. Además, brinda la oportunidad de la organización personal del tiempo, promoviendo la autorregulación, por lo que se trata de una categoría que bien merece la pena ser desarrollada y promovida entre los educadores de la EGB.

La sétima categoría, sustituto de la acción docente (SAD), obtiene solo dos evidencias (4\%) entre el total de 47 participantes del estudio. Las respuestas vinculadas a esta se clasifican en dos sub categorías: "construcción de recursos digitales autosuficientes" y "uso de recursos en línea autosuficientes". Ambas presentan una frecuencia muy baja: una para cada subcategoría. De ahí, se interpreta que los docentes poseen un escaso repertorio de acciones didácticas que les permitan emplear las TIC en este sentido, lo cual sería también objeto de atención, pues su empleo da la posibilidad de establecer interacciones de carácter reflexivo y cooperativo en torno a los contenidos de los materiales educativos trabajados.

Así, para el uso de recursos en línea autosuficientes, solo se hizo una relación con la página www.esl-lab.com. Esta página web consiste en un laboratorio para profesores y estudiantes. Con respecto a los profesores, les da la oportunidad de acceder a material para el aprendizaje del inglés en las destrezas de escucha y gramática; y el profesor puede ofrecer ejercicios para que los estudiantes los hagan dentro del aula o también, puede sugerir la página web para que ellos mismos naveguen y practiquen gramática o escucha. Por su parte, para la construcción de recursos digitales autosuficientes, se dio una respuesta que enfatiza en el uso de la herramienta EdiLIM para la construcción de libros interactivos. EdiLIM forma parte del sistema LIM, un entorno para la creación de materiales educativos. Este presenta un editor de actividades, un visualizador y un archivo en formato XML, el cual define lo que será el libro y sus páginas. Entre sus ventajas se cuenta que es innecesario instalar el programa en la computadora, su acceso es inmediato, no depende de un sistema operativo específico y es un entorno abierto. 
De la misma manera que en la categoría anterior, llama la atención que el registro de acciones y usos cotidianos sea tan baja, ya que en la web existe una serie de recursos que se convierten en herramientas digitales autosuficientes. Así, aparte de EdiLIM, se tienen EXeLearning, EclipseCrossword, Hot Potatoes, entre otras, que le permiten al docente diseñar actividades de aprendizaje y módulos de trabajo que propician la interacción, el trabajo en equipo y el desarrollo progresivo de la autorregulación y la autonomía en el aprendizaje.

Así las cosas, en materia de esta categoría es mucho el trabajo de capacitación y actualización docente que puede desarrollarse.

Para la categoría instrumentos de evaluación de los procesos de enseñanza aprendizaje (IEPEA), solo se encontró un dato que manifiesta correspondencia, evidenciado por el siguiente comentario: "...con los libros de autoevaluación los estudiantes generan actividades (digitales) en grupo e individuales en donde ponen en práctica todas sus destrezas en el idioma" (GVP, 2011, Dirección Regional Educativa de Los Santos, Primaria, Ficha Técnica, pregunta 3).

El proceso de autoevaluación, en este caso, se realiza en el área de inglés, para ello, los estudiantes hacen uso de unos libros que el docente denomina de autoevaluación. Estos libros, con base en la ficha técnica de la innovación, suponen una serie de ejercicios que permiten que el estudiante ponga a prueba sus conocimientos y, a la vez, sea partícipe de la valoración del proceso.

Una opción metodológica interesante para favorecer procesos de autoevaluación que promuevan la autorregulación del aprendizaje son los auto informes, en los cuales se consigna detalladamente la forma en que un aprendiz ha concebido y desarrollado una tarea académica compleja en sus distintas fases. En este tipo de autoevaluación de carácter reflexivo suelen desarrollarse habilidades introspectivas y metacognitivas que facilitan el desarrollo de la autorregulación y la autonomía en el aprendizaje.

Llama la atención que, siendo la evaluación un proceso vital en espacios de enseñanza y aprendizaje, haya tenido tan pocos aportes en las innovaciones realizadas por docentes de Educación General Básica, aun cuando sus potencialidades saltan a la vista. Quizá los anteriores datos sugieren la posibilidad de que el maestro de planta posee escasos recursos didácticos para implementar estrategias de autoevaluación a través del uso de las TIC.

Asimismo, la categoría instrumentos de evaluación de los resultados del aprendizaje (IERA) consigna un único dato ligado a la evaluación de productos por medio de la creación de recursos multimedia. Dado que la evaluación -sobre todo la enfocada en el proceso- es cambiante y, además, debe ser encarada de nuevas formas, las TIC proporcionan herramientas en las que el docente puede apoyarse para el desarrollo de una evaluación de carácter constructivista, procesual y auténtica. Así, estas pueden emplearse en procesos de evaluación de varias formas; entre ellas se señala que el estudiante tiene la posibilidad de crear objetos de evaluación: creación de textos cooperativos (en Google Docs y Wikispaces), presentaciones o cualquier tipo de material digital que se conciba como una evidencia del aprendizaje alcanzado a lo largo de una secuencia didáctica. 
URL: http://www.una.ac.cr/educare

CORREO: educare@una.cr

Otra manera es que la información le sea enviada al profesor o se comparta mediante recursos Web 2.0. Esto favorece una evaluación continua de manera formativa conforme el maestro realice el seguimiento, y quien aprende corrige o mejora lo mencionado. Además, promueve procesos de coevaluación, pues se facilita el que todos los participantes del proceso realimenten el trabajo.

Igualmente, el uso de recursos de audio y video, la manipulación de imágenes, la resolución de ejercicios mediante software, el trabajo cooperativo y los chats, son otras herramientas valiosas para la promoción de espacios de evaluación (autoevaluación, coevaluación, entre otros). Es la creatividad del docente y su habilidad para integrar los contenidos curriculares con el tipo de actividad que desea diseñar, las que a la postre le permitirán constatar el nivel de logro de sus estudiantes.

La décima categoría, producción de objetos tecnológicos con tecnología (POTT), obtiene un total de 10 inclusiones del total de participantes (21\%). La subcategoría de producción de tecnologías para la resolución de problemas, reporta el 50\% de los usos, al propiciar espacios donde los estudiantes tienen que aprender a investigar, diseñar y programar con software especializado para la robótica, fomentan la creatividad y una producción basada en la resolución de problemas contextualizados a su entorno.

Para la subcategoría, diseño de recursos didácticos para favorecer el aprendizaje, el otro $50 \%$ refiere que los estudiantes crean recursos como juegos programados, libros interactivos y multimedios. Al producir estos materiales, los estudiantes pueden practicar diferentes contenidos construyendo y compartiendo sus propios ejercicios. Además, los docentes participantes indican que propiciar un ambiente para la creación de recursos permite que sus alumnos adquieran habilidades para organizar una presentación en la que exponen todos aquellos aprendizajes que hayan adquirido durante el proceso.

Para la decimoprimera categoría, fortalecimiento de los aspectos emocionales (ASE), hay evidencias de 38 participantes, lo que corresponde a $81 \%$ de los datos generales, el número más amplio entre todas las categorías indagadas. Con base en los datos aportados por los participantes, se logró identificar que el 66\% (25) de los docentes que han utilizado las TIC lo hacen para fortalecer aspectos de carácter motivacional en los estudiantes. El desarrollo de habilidades para la vida se ubica en segundo puesto en relación con las demás frecuencias, con un $21 \%$ (8) de los datos, porcentualmente muy por debajo de la subcategoría referente al desarrollo de aspectos de carácter motivacional, con una diferencia de $45 \%$. Por último, se encuentra la subcategoría promoción componentes del proceso de autoestima, la cual está ubicada en tercer lugar, con una frecuencia del 13\% (5 participantes).

Al valorar la información aportada por los docentes participantes en este estudio, se evidencia que la motivación se da de dos formas: la primera remite directamente a la que genera en los estudiantes el uso de las TIC en los procesos de mediación que realiza el docente; y la segunda se refiere directamente a la que surge en el estudiante ante el uso de alguna de las herramientas tecnológicas. La primera forma de motivación, esto es, la de mayor peso dentro de esta subcategoría es el fortalecimiento de aspectos de carácter emocional. 
En el primero de los casos, los docentes indican que a través de actividades y proyectos asociados al uso de las TIC se genera en el estudiantado motivación, interés, gusto por la actividad - lección; pero, además, indican algunas características asociadas, como que facilita el abordaje de los contenidos temáticos, la disciplina, la dinámica y la interacción durante las lecciones. En segundo lugar, los docentes manifiestan que en los estudiantes se genera motivación, entusiasmo e interés con el uso de las TIC, pues les es fácil utilizar este tipo de herramientas, les permite tener acceso a recursos de los que carecen en sus hogares y les despierta la creatividad; produciendo mayor empeño en sus proyectos, una mejor puntualidad y un aumento en el dinamismo en las clases.

Es relevante destacar que no solo se trata del uso de recursos tecnológicos para lograr estudiantes interesados y motivados; no es una fórmula mágica, sino que la labor didáctica del docente tiene una huella ineludible, tal como lo indica Romero (2012) cuando resalta el papel del contexto como un factor que activa el interés y motivación por aprender. La mediación docente a través del uso de herramientas tecnológicas permite mayor identificación con la tarea de aprendizaje propuesta, contribuyendo paralelamente a la implicación emocional del estudiante con su aprendizaje; especialmente si esta mediación cuenta con una intencionalidad docente. Se cambia así el entorno de comunicación, ya que el estudiante emplea recursos que por lo general utiliza en su tiempo de ocio y esparcimiento. De este modo, el uso de las TIC no se presenta en abstracto, sino que contiene elementos del contexto, los cuales pueden favorecer el interés y motivación de los estudiantes.

Para este estudio, se colige que la motivación es un elemento importante en los procesos educativos, y es posible que las TIC que se utilizan en el salón de clase sean un factor relevante para generarla entre los estudiantes. Según los datos aportados por los docentes, se favorece la motivación a través de las actividades planificadas con las TIC, que dan como resultado la motivación; entre dichas actividades destacan los proyectos educativos, los cancioneros, los foros, los videos y los programas computacionales como el PowerPoint.

Con respecto al abordaje de temáticas con contenidos sociales, se destaca el desarrollo de temas que favorezcan la creación de mensajes positivos por parte de los estudiantes para el fomento de valores; además, se desarrollan temáticas relacionadas con sexualidad, embarazo adolescente, maternidad y paternidad responsable, toma de decisiones y proyecto de vida.

Sobresale que el fortalecimiento de esas habilidades no fue de carácter intencional, pues en ninguno de los casos se indica que este fuera el verdadero cometido, sino que surge como consecuencia del uso de las TIC en el aula con fines académicos, y no específicamente enfocados hacia el fortalecimiento de habilidades para la vida.

Los datos que tratan sobre el abordaje de temáticas con contenidos sociales tienen la intencionalidad de tratar estos contenidos con los estudiantes y se enfocan en gran medida en las habilidades cognitivas; a diferencia de los otros datos, que se relacionan con el desarrollo de habilidades sociales. 
URL: http://www.una.ac.cr/educare

CORREO: educare@una.cr

Las TIC pueden potenciar procesos de fortalecimiento de la autoestima a través de la promoción de sus componentes, beneficiando el desarrollo integral de los estudiantes. Esto gracias a que disparan elementos que le permiten al estudiante autoconocerse, descubrir capacidades, motivarse y desarrollar comportamientos flexibles y ajustados al contexto, lo cual favorece no solo elementos para la autoestima personal y el desarrollo integral del estudiantado, sino también para formar un autoconcepto académico y enfrentar de una mejor manera su proceso de aprendizaje.

Finalmente, los datos analizados en este apartado permiten visualizar las TIC como herramientas flexibles en el sentido de que le posibilitan al personal docente favorecer diversas áreas personales y académicas de sus estudiantes.

La décimo segunda y última categoría, potenciadores de las capacidades de aprendizaje $(P C A)$, muestra una predominancia de elementos vinculados a la subcategoría "desarrollo del aprendizaje significativo", en la cual existen un total de 8 respuestas vinculadas; lo que representa un $42,2 \%$ del total.

Por otra parte se encuentra la subcategoría "desarrollo del pensamiento estratégico", la cual contabiliza 5 respuestas, es decir, un $26,3 \%$ de las vinculadas a esta categoría. En tercer lugar el gráfico muestra con un total de 4 respuestas para la subcategoría "desarrollo del pensamiento creativo", un $21 \%$ del total; y por último, con un total de 2 respuestas, aparece las subcategoría "desarrollo del pensamiento lógico matemático, la cual representa un 10,5\% del total de respuestas consignadas.

En síntesis, los datos encontrados indican que los docentes participantes en este estudio emplean las TIC con mayor frecuencia como "elementos potenciadores del aprendizaje", a través de estrategias didácticas que fomenten el desarrollo el aprendizaje significativo.

Por potenciadores de las capacidades de aprendizaje se han definido las estrategias didácticas con el uso de TIC que, de acuerdo con Salomón (1992), de manera consciente o inconsciente, promueven el desarrollo de capacidades de aprendizaje. Para el presente estudio, entendemos por capacidades de aprendizaje las siguientes: desarrollo de la creatividad, aprendizaje significativo, pensamiento estratégico y pensamiento lógicomatemático.

Algunos de los indicadores que revelan los datos analizados son los siguientes: estimulación de creatividad e imaginación, desarrollo de la creatividad e independencia académica, potenciación del aprendizaje significativo, auto reflexión, desarrollo de habilidades lógicomatemáticas y apropiación de los procesos de aprendizaje en la construcción de libros interactivos, entre otros.

En este sentido, se identifican indicadores que se agrupan en 5 subcategorías, entre las cuales se destacan: desarrollo de la creatividad, desarrollo del aprendizaje significativo, desarrollo del conocimiento estratégico y del pensamiento lógico-matemático. 
Los datos revelados en el presente estudio señalan que el estudiantado, al trabajar con tecnologías, desarrollan autonomía y habilidades de auto aprendizaje, lo cual sin duda favorece el desarrollo de la autorregulación y el conocimiento estratégico. Las TIC, por las características de los diversos entornos, software y plataformas que emplean, representan un instrumento para favorecer estrategias de enseñanza y de aprendizaje que fomenten un uso consciente del conocimiento estratégico, lo cual tendrá un impacto seguro en la formación de individuos capaces de aprender a lo largo de la vida y de transferir lo aprendido a diversas situaciones y contextos.

\section{Las conclusiones}

Con base en las evidencias y su análisis, se considera que las prácticas didácticas mediadas con TIC por personal docente de EGB corresponden a las siguientes:

1. Actividades y proyectos con el uso de TIC, que simultáneamente desarrollaron la motivación y el interés del estudiantado.

2. Manejo básico de dispositivos de entrada y salida por parte de docentes y estudiantes: cámaras fotográficas y de video, teléfonos móviles y video beam.

3. Uso del internet: comunicación, búsqueda de información e imágenes y recursos en la web 2.0. Según los datos del estudio, esto implica la selección, valoración, análisis o síntesis de la información encontrada para el desarrollo de actividades en la clase, tales como: creación de diapositivas, exposiciones, resúmenes, investigaciones, análisis, uso del Facebook, blogs, chats y foros.

4. Utilización de software, aplicaciones y herramientas: Robolab, Prezi, Edilim, Scratch, fotos narradas, Movie Maker y Microsoft PowerPoint.

5. El uso de las TIC como un recurso para apoyar procesos de evaluación de los aprendizajes resulta poco significativo en la práctica.

Igualmente, entre los usos más destacados que los docentes hicieron de las TIC en las prácticas educativas señaladas sobresale la categoría emergente denominada "aspectos emocionales" (ASE), como la que representa mayor predominancia. Lo anterior permite afirmar que el personal docente posee claridad en cuanto a que la educación debe estar vinculada a aspectos motivacionales para generar aprendizajes duraderos y significativos.

En segundo lugar, las respuestas obtenidas presentaron una relación estrechamente vinculada a la categoría denominada "contenido de aprendizaje". El personal docente participante considera importante enseñar aspectos de carácter técnico relacionados con el uso 
URL: http://www.una.ac.cr/educare

CORREO: educare@una.cr

de las herramientas tecnológicas como contenidos durante su práctica pedagógica, a pesar de no estar contemplados en los programas de estudio.

En tercer lugar, se destaca la categoría denominada "potenciadores de las capacidades de aprendizaje". Esta muestra la predominancia de prácticas educativas relacionadas con el desarrollo del aprendizaje significativo, desarrollo del pensamiento estratégico, desarrollo del pensamiento creativo y desarrollo del pensamiento lógico matemático. Los datos evidencian que el uso estratégico de las TIC por parte de los docentes participantes, conlleva al desarrollo paralelo de tipos de andamiajes que favorecen los aspectos anteriormente mencionados.

Destacadas en el quinto lugar se encuentran las categorías "herramientas de búsqueda y selección de los contenidos de aprendizaje" y "herramientas de comunicación entre los participantes". La primera se refiere a la indagación y selección de contenidos de aprendizaje utilizando las TIC en los procesos de enseñanza y aprendizaje. Los docentes participantes propician la búsqueda y selección de los contenidos que se descargan de la web como insumo para su labor docente. En lo que compete a la segunda categoría, se concluye que las prácticas didácticas más frecuentes son las relacionadas con la orientación comunicativa de las herramientas Web 2.0 y a través de dispositivos móviles.

En quinto lugar, sobresale la categoría "auxiliares o amplificadores de la acción docente" en la cual se concluye que los docentes participantes utilizan recursos multimedia, software educativo y herramientas en línea con el propósito de dinamizar la práctica pedagógica y potenciar los aprendizajes.

Finalmente, concluimos que el análisis de la información nos ha permitido contrastar cuáles son los usos de las TIC y sus atribuciones didácticas en la EGB, por lo menos para los usos que mayor frecuencia reportaron. A partir de ello tenemos:

Respecto a la categoría relacionada con "aspectos emocionales", se evidencia que la motivación se presenta en dos vías: extrínseca e intrínseca. La primera es generada intencionalmente por el docente al hacer un uso atractivo de las herramientas tecnológicas. En la segunda, el hecho de trabajar con TIC hace que el estudiante desarrolle la motivación que le permite autorregular su aprendizaje.

En cuanto a la categoría vinculada con el uso de las TIC como "contenido de aprendizaje", se concluye que es importante para los docentes la inclusión de espacios específicos en el planeamiento de aula, los cuales apunten hacia la enseñanza explícita de elementos relacionados con el uso de hardware, tales como: mecanismo de encendido del equipo, dispositivos de entrada y de salida, uso de cámaras de video y digitales, GPS, teléfonos celulares, algunos motores, sensores y engranajes. 
Por otra parte, y con respecto a la categoría "potenciadores de las capacidades de aprendizaje", se tiene evidencia de que esta representa la siguiente categoría más empleada por los docentes participantes. Esta se establece a través de estrategias didácticas que fomenten el desarrollo del aprendizaje significativo, la creatividad, el pensamiento estratégico y el razonamiento lógico.

En lo referente a la categoría "auxiliar o amplificador de la acción docente", se establece que los educadores participantes emplean el proyector multimedia para mostrarles a los estudiantes videos y fotos. Además, lo usan para ver películas, documentales o videos; también utilizan el Movie Maker, EdiLim y PowerPoint para presentaciones, y en menor medida como software educativo o como herramienta en línea.

En cuanto a la categoría denominada "herramientas de búsqueda y selección de los contenidos de aprendizaje", se enfatiza la búsqueda de información con el uso de las TIC, la cual se vale de herramientas tales como la red internet, dispositivos móviles y celulares.

En lo referente a la categoría "herramientas de comunicación entre los participantes", las múltiples posibilidades didácticas e interactivas que las TIC ofrecen representan una plataforma de comunicación apta para el desarrollo de espacios de diálogo que configuren nuevas formas de enseñanza y aprendizaje.

De acuerdo con la categoría "instrumentos cognitivos a disposición de los participantes", se señala que el uso de software multimedia es utilizado por el personal docente como herramientas de mediación entre los contenidos y el aprendizaje.

En cuanto a la categoría denominada "producción de objetos tecnológicos con tecnología", las experiencias en donde se emplea la robótica como eje conductor de procesos de diseño y producción de objetos tecnológicos de cara a la resolución de problemas particulares, provoca que el estudiantado incremente su potencial creativo, expresivo y cognoscitivo, al evaluar, reflexionar y resolver problemas concretos a partir del uso de la tecnología.

\section{Referencias}

Bustos, A. y Coll, C. (enero-marzo, 2010). Los entornos virtuales como espacios de enseñanza y aprendizaje. Una perspectiva psicoeducativa para su caracterización y análisis. Revista mexicana de investigación educativa, 15(44), 163-184. Recuperado de http://www.redalyc. org/pdf/140/14012513009.pdf 
URL: http://www.una.ac.cr/educare

CORREO: educare@una.cr

Claro, M. (2010). La incorporación de tecnologías digitales en educación. Modelos de identificación de buenas prácticas. Santiago: CEPAL. Recuperado de http://www.eclac.org/publicaciones/ $\mathrm{xml} / 8 / 40278 /$ tics-educacion-buenas-practicas.pdf

Coll, C. (s. f.). Las TIC en el aula. Aprender y enseñar con las TIC: Expectativas, realidad y potencialidades. En R. Carneiro, J. C. Toscano y T. Díaz (Coords.), Los desafíos de las TIC para el cambio educativo (pp. 111-126). Madrid: OEl y Fundación Santillana. Recuperado de http://www.educando.edu.do/files/6613/7875/6220/Los desafios delas TIC para elcambio educativo.pdf

Coll, C. (agosto-enero, 2004). Psicología de la educación y prácticas educativas mediadas por las tecnologías de la información y la comunicación. Una mirada constructivista. Revista Electrónica Sinéctica, 25, 1-24. Recuperado de https://docs.google.com/viewer?url=http:// www.redalyc.org/pdf/998/99815899016.pdf\&chrome=true

Coll, C., Mauri, T. y Onrubia, J. (2008). La utilización de las tecnologías de la información y la comunicación en la educación: Del diseño tecno-pedagógico a las prácticas de uso. En C. Coll, \& C. Monereo (Eds.), Psicología de la educación virtual. Aprender y enseñar con las tecnologías de la información y la comunicación (pp. 74-103). Barcelona: Ediciones Morata.

Lugo, M. T. (2010). Las políticas TIC en la educación de América Latina. Tendencias y experiencias. Revista Fuentes, 10,52-68. Recuperado de http://www.oei.es/noticias/spip.php?article9720

Monereo, C., Castelló, M. y Gómez, I. (2009). La evaluación como herramienta de cambio educativo: Evaluar las evaluaciones. En C. Monereo (Coord.), Pisa como excusa. Repensar la evaluación para cambiar la enseñanza (pp. 16-33). Barcelona: Grao. Recuperado de http:// www.carlesmonereo.com/wp-content/uploads/2012/09/MONEREO-C.-coord.-2009-.pdf

Monereo, C. y Romero, M. (2008). Los entornos virtuales de aprendizaje basados en sistemas de emulación socio-cognitiva. En C. Coll y C. Monereo (Eds.), Psicología de la educación virtual. Aprender y enseñar con las tecnologías de la información y la comunicación (pp. 194-212). Barcelona: Ediciones Morata.

Pérez-González, J. C. (2012) Revisión del aprendizaje social y emocional en el mundo. En R. Bisquerra (Coord.), ¿Cómo educar las emociones? La inteligencia emocional en la infancia y la adolescencia (pp. 56-69). Barcelona: Hospital Sant Joan de Déu. Recuperado de http:// faros.hsjdbcn.org/sites/default/files/faros 6 cast.pdf

Picardo, O. (mayo-agosto, 2002). Pedagogía informacional. Enseñar a aprender en la sociedad del conocimiento. Revista Iberoamericana de Ciencia, Tecnología, Sociedad e innovación, 3. Recuperado de http://dialnet.unirioja.es/servlet/articulo?codigo=741449

Pozo, J. I., Monereo, C. y Castelló, M. (2001). La enseñanza de estrategias de aprendizaje en el contexto escolar. En C. Coll, J. Palacios y Á. Marchesi, Desarrollo psicológico y educación (Vol. 2). Psicología de la educación escolar (pp. 235-258). Madrid: Alianza. 
Romero, M. D. (abril-junio, 2012). Uso de las tecnologías de la información y la comunicación para la gestión del conocimiento en el proceso de enseñanza-aprendizaje. Revista Didasc@Lia: Didáctica y Educación, 3(2), 1-16. Recuperado de http://revistas.ojs.es/index. php/didascalia/issue/view/68/showToc

Salinas, J. (julio, 1997). Nuevos ambientes de a aprendizaje para una sociedad de la información. Revista Pensamiento Educativo, 20, 81-104. Recuperado de http://www.ses.unam.mx/ curso2008/pdf/Salinas.pdf

\section{Cómo citar este artículo en APA:}

Campos, K., Carvajal, V., Castro, E. R., Hutchinson, S., Masís, M., Murillo, S., Rojas, G., ... y Sánchez, G. (enero-abril, 2014). Actividades de aprendizaje y TIC: Usos entre docentes de la Educación General Básica costarricense. Aproximación diagnóstica. Revista Electrónica Educare, 18(1), 239263. Recuperado de http://www.revistas.una.ac.cr/index.php/EDUCARE/issue/current

Nota: Para citar este artículo en otros sistemas puede consultar el hipervínculo "Como citar el artículo" en la barra derecha de nuestro sitio web:

http://www.revistas.una.ac.cr/index.php/EDUCARE/index 